\title{
Automatic Vehicle Tracking System Based on Inductance Sensor
}

\author{
Lijian Cai $^{1, a}$, Yecai Guo ${ }^{1,2, b^{*}}$ and Xiang Wang ${ }^{1, c}$ \\ ${ }^{1}$ School of Electronic and information Engineering , Nanjing University of Information Science and \\ Technology, Nanjing210044, China) \\ ${ }^{2}$ Collaborative Innovation Center of Atmospheric Environment and Equipment Technology, Nanjing \\ 210044, China) \\ a314759180@qq.com, bguo-yecai@163.com, '157691455@qq.com
}

Keywords: STM32; Intelligent vehicle; Inductive sensor; Variable pulse signals

\begin{abstract}
Automatic vehicle tracking system based on inductance sensor was designed. This system uses inductive sensor for data acquisition controlled by STM32 microcontroller. The vehicle is made up of L298N motor drive module, geared motor module, buzzer sound module, LCD module, encoder module, bluetooth module, and battery module, and in order to change direction and speed by DC motor, its four-wheel drive vehicle can output variable pulse signals. Microcontroller continually scans four-channel data of LDC1614 and controls vehicle to complete tracing action. If an obstacle is detected by it, the buzzer module will make a sound prompts. The precise timing can be obtained by the timer module, and the encoder module is designed to measure the speed of the vehicle. The designed system can implement the function of real-time display of time and distances, and has high precision and low power consumption.
\end{abstract}

\section{Introduction}

In recent years, intelligent vehicles have become hot issue in the world's vehicle engineering research and new impetus of the automotive industry and many developed countries have placed special focus on the development of intelligent transportation system. At present, modern vehicle tracking systems commonly use photoelectric sensor, digital camera, etc. However, these technologies have great dependence on external environmental conditions and cannot avoid being influenced by light and weather conditions for the limitation of sensor's working environment.

In this paper, a new intelligent tracking vehicle system based on electromagnetic induction principle is proposed. Under the protection of the electromagnetic shielding system, the inductive sensor will not be affected by the weather and some complicated conditions. Thus, the electromagnetic navigation has better anti-interference ability to the external factors than the light-based or image-based navigation. Moreover, a new algorithm for data processing and a new coil placement method have been proposed. After adjusting the position of the coil, the mutual inductance happens between coil and track. The microcontroller can locate the position of vehicle via using a specific algorithm to process the sensor data in order to control motor for the next step of the movement. Consequently, the goal of intelligent vehicle tracking has been realized. In addition, microcontroller can judge and process the sensor data quickly and effectively, to accurately grasp the effective data and remove the unnecessary interference items, so that this process of tacking meets the requirement of the accurate location.

\section{Target Design and Plan Selection}

Target Design. Our goal is to implement high-precision automatic tracking function based on inductive sensors and to use a new algorithm to process different data of six coils, so as to acquire the precise location of vehicle for automatic control. What's more, the vehicle can judge and voice prompts if it detects obstacles by buzzer module and display real-time speed and travel distance on the LCD screen. 
Overall Plan Selection. Overall plan is shown in Fig. 1.

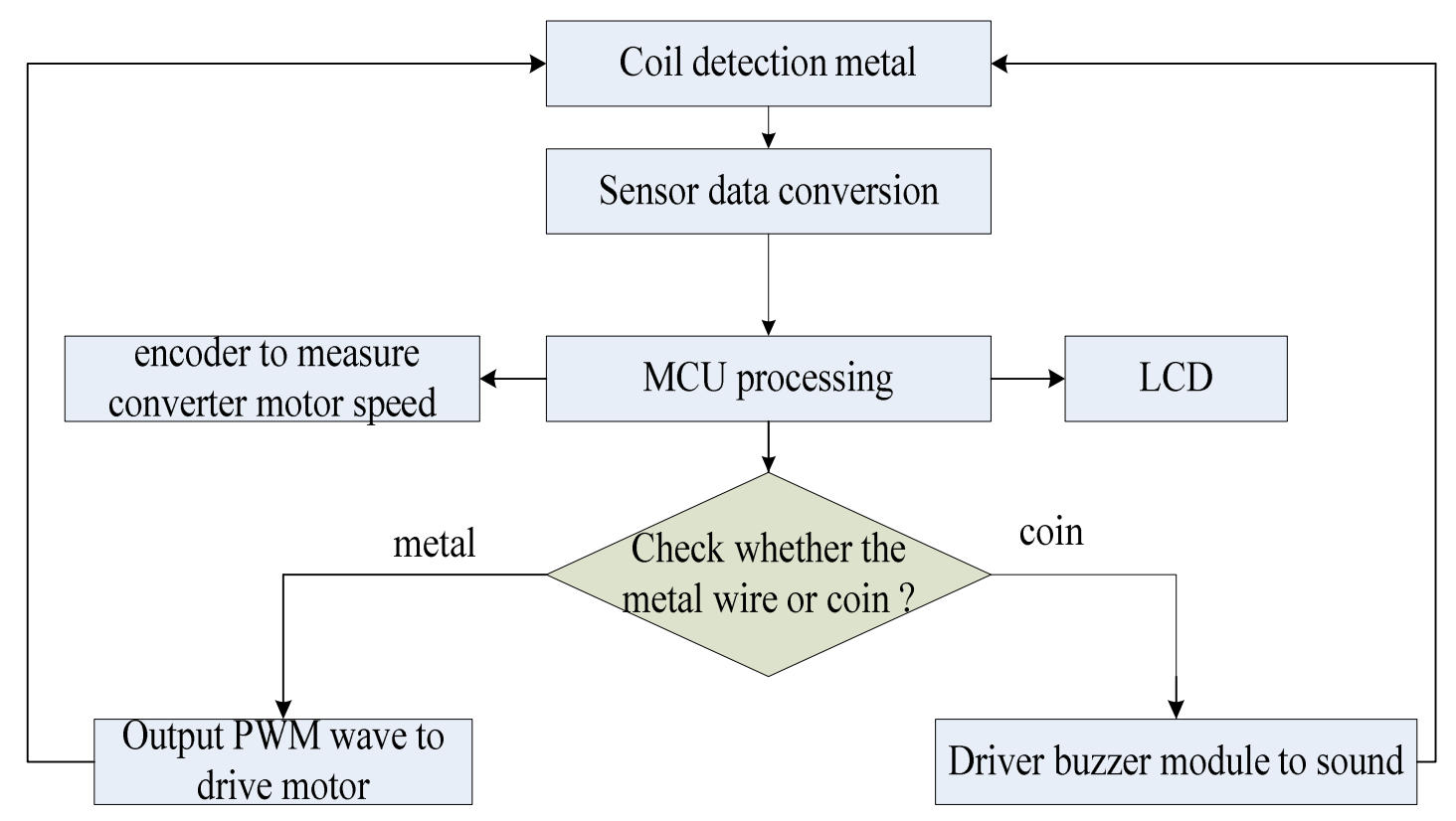

Figure 1. Overall Plan

The design uses digital inductor converter as a detector to detect the iron trace for data acquisition, which can be processed by microcontroller and then output variable periodic pulse signal to drive slowdown DC motor in order to implement steering control and change vehicle speed .When the sensor detects obstacles, microcontroller would transmit a signal to make buzzer module voice prompts. In the entire journey of the vehicle, the precise timing is realized by the timer module and the real-time running time can be displayed on the LCD. Microcontroller reads pulse signal numbers in unit time to measure the wheel speed and display travel distance on the screen[1].

\section{Hardware Selection and Circuit Design}

Microcontroller. In consideration of tracking vehicle's size and function, we use STM32F103RC of 64Pin chip as the microcontroller with 32-bit RISC core operating at a $72 \mathrm{MHz}$ frequency, high-speed embedded memories(Flash memory up to 32 Kbytes and SRAM up to 6 Kbytes), and an extensive range of enhanced I/Os and peripherals connected to two APB buses. The devices offer two 12-bit ADCs, three general purpose 16-bit timers plus one PWM timer, as well as standard and advanced communication interfaces: up to two I2Cs and SPIs, three USARTs, an USB and a CAN.

Inductive Sensor. The LDC1614 as inductive sensor is 4-channel, 28-bit inductance to digital converters (LDCs) for inductive sensing solutions[2] with multiple channels and support for remote sensing. It is easy to use, only requiring that the sensor frequency be within $1 \mathrm{kHz}$ and $10 \mathrm{MHz}$ to begin sensing. The wide $1 \mathrm{kHz}$ to $10 \mathrm{MHz}$ sensor frequency range also enables use of very small PCB coils, further reducing sensing solution cost and size. The high resolution channels allow for a much larger sensing range and maintain good performance beyond two coil diameters. Well-matched channels allow for differential and ratiometric measurements. In this system, we use it for data acquisition, which can be processed by microcontroller. In addition, the advanced-control timer(TIM1)[3] can be seen as a three-phase PWM multiplexed on 6 channels. It has complementary PWM outputs with programmable inserted dead-times. It can also be seen as a complete general-purpose timer. The 4 independent channels can be used for input capture, output compare, PWM generation (edge- or center-aligned modes), and one-pulse mode output.

Motor Drive and Encoder. The L298N, used as motor drive module, is an integrated monolithic circuit in a 15-lead Multiwatt and PowerSO20 packages. It is a high voltage, high current dual 
full-bridge driver designed to accept standard TTL logic levels and drive inductive loads such as relays, solenoids, DC and stepping motors. Two enable inputs are provided to enable or disable the device independently of the input signals. The emitters of the lower transistors of each bridge are connected together and the corresponding external terminal can be used for the connection of an external sensing resistor. An additional supply input is provided so that the logic works at a lower voltage.

GP1A038RBKL, used as encoder module, is a linear encoder for reading linear scale. Moreover, since the multi-divided photodiode system is adopted, high precision reading is possible even if the angle is deviated between the scale and encoder.

Display. SSD1289 TFT Driver is an all in one driver that integrated the RAM, power circuits, gate driver and source driver into single chip. It can drive a $262 \mathrm{k}$ color a-TFT panel with resolution of 240 RGB x 320.It also integrated the controller function and consists of up to 172,800 bytes (240 x 320 x 18 / 8) Graphic Display Data RAM (GDDRAM) such that it interfaced with common MCU through 8/9/16/18-bits 6800-series /8080-series compatible Parallel Interface or Serial Interface and stored the data in the GDDRAM. Auxiliary18-bits video interface(VSYNC, HSYNC, DOTCLK, ENABLE)are integrated into SSD1289 for displays animated image. SSD1289 embeds DC-DC Converter and Voltage generator to provide all necessary voltage required by the driver with minimum external components. A Common Voltage Generation Circuit is included to drive the TFT-display counter electrode. An Integrated Gamma Control Circuit is also included that can be adjusted by software commands to provide maximum flexibility and optimal display quality. SSD1289 can be operated down to $1.16 \mathrm{~V}$ and provide different power save modes. It is suitable for any portable battery-driven applications requiring long operation period with compact size.

\section{Software Design}

LDC1614 Communication. I2C-bus gives a wide range of benefits to equipment manufacturers based on the following reasons: (1) The simple 2-wire serial I2C-bus minimizes interconnections so ICs have fewer pins and there are not so many PCB tracks; result-smaller and less expensive PCBs. (2) The completely integrated I2C-bus protocol eliminates the need of addressing decoders and other glue logic. (3) The multi-master capability of the I2C-bus allows rapid testing and alignment of end-user equipment via external connections to an assembly-line. (4) The availability of I2C-bus compatible ICs in SO (small outline),VSO (very small outline) as well as DIL packages reduces space requirements even more. After initialization of the system and IIC,LDC1614 register will be written different values that contribute to sensor for working[5][6].Due to the IIC bus, the microcontroller can read data continually.

Timer Design. There are two synchronizable general-purpose timers embedded in the STM32 F103xx performance line devices. These timers are based on a 16-bit auto-reload up/down counter, a 16-bit prescaler and feature 4 independent channels each for input capture/output compare, PWM or one-pulse mode output. This gives up to 12 input captures/output compares/PWMs on the largest packages. The general-purpose timers can work together with the advanced-control timer via the Timer Link feature for synchronization or event chaining. Their counter can be frozen in debug mode. Any of the general-purpose timers can be used to generate PWM outputs. They all have independent DMA request generation[7]. In this system, we use CH1 of the TIM2 as input capture and $\mathrm{CH} 2$ as output compare. We can precisely measure operating time of vehicle via capturing and comparing[8].

\section{Tracking Algorithm}

Placement of Coins. In order to keep the speed that we want during the tracking, a type of placement for forward-looking and obstacle-detecting were designed. In the forward-looking, we place three coils, as shown in Fig. 4(a). In the obstacle-detecting (coins), we place another there coins in the front three coils[9], as shown in Fig. 2 (b). 


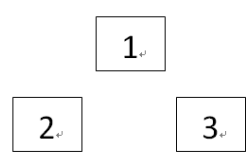

(a)

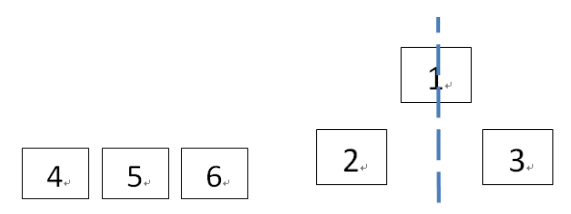

(b)

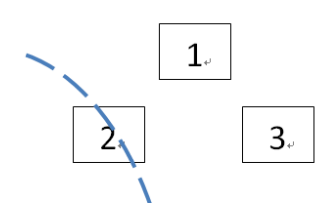

(d)

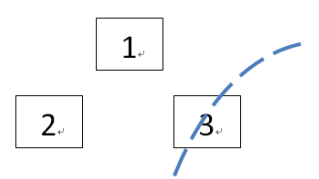

(e)

Figure 2. Tracking of the vehicle

Detect Obstacles Design. LDC1614 four channels are all used for data acquisition, based on IIC bus. Three channels are used for three coins separately and one channel is used for the front three coils which are connected with each other. Since the range of value of feedback from sensor is set due to the feature of the two materials, based on electromagnetic induction[10], we set 5 as the standard of judgement, according to lots of tests. If the value is greater than 5 , we judge that the vehicle has discovered obstacle. By contrast, if the value is smaller than 5, we judge that there is no obstacle and we can start tracking. In the process of system's running, four channels works simultaneously for data acquisition and immediately sent the data to microcontroller for voicing prompt and controlling motor[11].

Tracking Algorithm. In order to implement the vehicle's straight and left or right turning, the proposed algorithm is given as follows:

Go Straight. When the No.1 coil is on the track, and No.2 and No.3 are not on the track, we judge that the vehicle is on the straight, when the microcontroller will let the vehicle go straight[12]. When the vehicle goes straight, microcontroller drive the motor to ensure that the four-wheel speed would maintain the same. Position is shown in Fig. 4(c).

Turn Left. When the No. 1 and No. 3 coils are outside the track, and No. 2 is on track at the same time, we judge that the vehicle must be approaching the left bend at the time, and the vehicle should turn left. When the vehicle turns left, microcontroller controls the left front and left rear wheels to stop working, and the right front and right rear wheels keep the previous rotating speed (the design has been tested correctly due to the track radius is nearly the same as the vehicle size)[13-14].As shown in Fig. 4(d)

Turn Right. When the No. 1 and No. 2 coils are outside the track, and No. 3 is on track at the same time, we judge that the vehicle must be approaching the right bend at the time, and the vehicle should turn right. When the vehicle turns right, microcontroller controls the right front and right rear wheels to stop working, and the left front and left rear wheels keep the previous rotating speed(the design has been tested correctly due to the track radius is nearly the same as the vehicle size). As shown in Fig. 4(e).

\section{Conclusion}

This vehicle, using inductive sensors, based on a new algorithm, can implement function of tracking, detection of obstacles, speed measuring, distance display and prompt voicing. Among lots of tests, the tracking nearly has no error. Late improvement can be implemented on hardware structure, on which we can adjust the number of the coil and the placement as well. It may meet the requirement of higher speed and accuracy, due to capacity of vehicle that deals with sudden change is improved.

\section{Acknowledgements}

This work is supported by the National Natural Science Foundation of China (61371131, 61673222), the Major Project of Nature Science Foundation of Higher Education Institution of Jiangsu Province, China(Grant No.13KJA510001), Jiangsu Scientific Research Achievements in Industrialization Project, China(JHB 2012-9), Jiangsu Province, and Top-notch Academic Programs Project of Jiangsu Higher Education Institutions(TAPP, PPZY2015B134). 


\section{Reference}

[1] Ahmed J, Jafri M N, Shah M, et al: Real-time Edge-enhanced Dynamic Correlation and Predictive Open-loop Car-following Control for Robust Tracking, Machine Vision and Applications, Vol. 19 (2008) No.1, p.1

[2] Daum F: Nonlinear filters: Beyond the Kalman Filter, Ieee Aerospace and Electronic Systems Magazine, Vol.20 (2005) No.8,p.57

[3] Petrella R and Tursini M: An Embedded System for Position and Speed Measurement Adopting Incremental Encoders, Ieee Transactions on Industry Applications, Vol. 44 (2008) No.5,p.1436

[4] Liu C and Dong Y: Resonant Enhancement of a Passive Coil-capacitance Loop in Eddy Current Sensing Path, Measurement, Vol. 45 (2012) No.3,p.622

[5] Dinulovic D and Gatzen H H: Microfabricated Inductive Micropositioning Sensor for Measurement of a Linear Movement, Ieee Sensors Journal, Vol. 6 (2006) No.6,p.1482

[6] Hespanha J P, Naghshtabrizi P and Xu Y: A Survey of Recent Results in Networked Control Systems, Proceedings of the Ieee, Vol. 95 (2007) No.1,p.138

[7] Sun Z H, Bebis G and Miller R: On-road Vehicle Detection: A Review, Ieee Transactions on Pattern Analysis and Machine Intelligence, Vol. 28 (2006) No.5,p.694

[8] Devasia S, Eleftheriou E and Moheimani S O R: A Survey of Control Issues in Nanopositioning, Ieee Transactions on Control Systems Technology, Vol. 15 (2007) No.5,p.802

[9] Misron N, Ying L Q, Firdaus R N, et al: Effect of Inductive Coil Shape on Sensing Performance of Linear Displacement Sensor Using Thin Inductive Coil and Pattern Guide, Sensors, Vol. 11 (2011) No.11,p.10522

[10] Cha Y-J, Nam B, Kim J, et al: Evaluation of the Planar Inductive Magnetic Field Sensors for Metallic Crack Detections, Sensors and Actuators a-Physical, Vol. 162 (2010) No.1,p.13

[11]Olfati-Saber R, Fax J A and Murray R M: Consensus and Cooperation in Networked Multi-agent Systems, Proceedings of the Ieee, Vol. 95 (2007) No.1,p.215

[12]Danisi A, Masi A, Losito R, et al: Electromagnetic Analysis and Validation of an Ironless Inductive Position Sensor, Ieee Transactions on Instrumentation and Measurement, Vol. 62 (2013) No.5,p.1267

[13] Wu B-F, Lin C-T and Chen Y-L: Dynamic Calibration and Occlusion Handling Algorithms for Lane Tracking, Ieee Transactions on Industrial Electronics, Vol. 56 (2009) No.5,p.1757

[14] Onieva E, Naranjo J E, Milanes V, et al: Automatic Lateral Control for Unmanned Vehicles via Genetic Algorithms, Applied Soft Computing, Vol. 11 (2011) No.1,p.1303 